Research Article

\title{
Second-Order Asymptotics of the Risk Concentration of a Portfolio with Deflated Risks
}

\author{
Yu Chen $(\mathbb{D}$, Yu Gao, Wenxue Gao, and Weiping Zhang \\ Department of Statistics and Finance, School of Management, University of Science and Technology of China, Hefei,
} Anhui 230026, China

Correspondence should be addressed to Weiping Zhang; zwp@ustc.edu.cn

Received 6 November 2017; Revised 15 January 2018; Accepted 21 February 2018; Published 17 April 2018

Academic Editor: Alessandro Arsie

Copyright (C) 2018 Yu Chen et al. This is an open access article distributed under the Creative Commons Attribution License, which permits unrestricted use, distribution, and reproduction in any medium, provided the original work is properly cited.

\begin{abstract}
The quantification of diversification benefits due to risk aggregation has received more attention in the recent literature. In this paper, we establish second-order asymptotics of the risk concentration based on several risk measures for a portfolio of $n$ identically distributed but dependent deflated risks $X_{j}=R_{j} S, j=1,2, \ldots, n$ under the assumptions of second-order regular variation on the survival functions of the risks $R_{j}$ and the deflator $S$, where $R_{1}, R_{2}, \ldots, R_{n}$ are $n$ independent and identically distributed random variables with a common survival function and $S$ is a random variable being independent of $R_{1}, R_{2}, \ldots, R_{n}$. Examples are also given to illustrate our main results.
\end{abstract}

\section{Introduction}

The quantification of diversification benefits due to risk aggregation plays a prominent role in the (regulatory) capital management of large firms within the financial industry. Measuring a risk and quantifying its diversification benefits have become an important task. Especially when the underlying risk factors show a heavy-tailed pattern, many papers discussed diversification benefits; see, for instance, Degen et al. [1] (2010), Ibragimov and Walden [2], Ibragimov et al. [3], Mao et al. [4], Lv et al. [5, 6], Hashorva et al. [7], and references therein.

Risk measure is understood as a function that can assign a nonnegative real number to a risk. Consider a portfolio of $n$ loss random variables $X_{1}, X_{2}, \ldots, X_{n}$. The risk concentration based on the risk measure $\varrho[\cdot]$ is defined as

$$
C_{\varrho}=\frac{\varrho\left[\sum_{i=1}^{n} X_{i}\right]}{\sum_{i=1}^{n} \varrho\left[X_{i}\right]} .
$$

Here, $1-C_{\rho}$ refers to the diversification benefit. In recent years, empirical work has argued that financial variables often exhibit stronger dependence, while the existing work usually assumes that the risks $X_{1}, X_{2}, \ldots, X_{n}$ are independent and identically distributed; see Embrechts et al. [8, 9], Degen et al. [10], Mao and $\mathrm{Hu}$ [11], Mao and $\mathrm{Hu}$ [12], Lv et al. [6], and so on. We focus on the asymptotic of risk concentration for a portfolio of $n$ identically distributed but correlated deflated risks $X_{j}=R_{j} S, j=1, \ldots, n$ under assumptions of secondorder regular variation on the survival functions of the risk $R_{1}, \ldots, R_{n}$ and deflator $S$.

In the present paper we study mathematical properties of diversification effects under the different risk measures $\varrho[\cdot]$. Several popular risk measures have been introduced to measure tail risk, such as the Value-at-Risk (VaR), the conditional tail expectation (CTE), and the HaezendonckGoovaerts risk measure. These risk measures have been used extensively in insurance and finance as a tool of risk management; see Denuit et al. [13], Artzner et al. [14], Cheung and Lo [15], Zhu et al. [16], and references therein. The Valueat-Risk (VaR) of $X$ at the level $p$ is defined as

$$
\operatorname{VaR}_{p}[X]=\inf \{x \in \mathbb{R}: F(x) \geq p\}, \quad p \in(0,1),
$$

and the conditional tail expectation (CTE) of $X$ at the level $p$ is defined as

$$
\mathrm{CTE}_{p}[X]=E\left[X \mid X>\operatorname{VaR}_{p}[X]\right], \quad p \in(0,1) .
$$

The Haezendonck-Goovaerts risk measure, which was introduced by Haezendonck and Goovaerts [17], is defined via an increasing and convex Young function $\phi$ and a 
parameter $p \in(0,1)$ representing the confidence level. More precisely, let $\phi$ be a nonnegative and convex function on $[0, \infty)$ with $\phi(0)=0, \phi(1)=1$, and $\phi(\infty)=\infty$. This function is called a normalized Young function. Assume we have a realvalued random variable $X$ with distribution function $F$ such that

$$
E[\phi(c X)]<\infty \quad \forall c>0,
$$

and let $H_{p}[X, t]$ be the unique solution $h$ to the equation

$$
E\left[\phi\left(\frac{(X-t)_{+}}{h}\right)\right]=1-p, \quad p \in(0,1),
$$

if $\bar{F}(t)>0$ and 0 if $\bar{F}(t)=0$, where $x_{+}=\max \{x, 0\}$ for any real number $x$. Then the Haezendonck-Goovaerts risk measure of $X$ at the confidence level $p$ is defined as

$$
\mathrm{HG}_{p}[X]=\inf _{t \in \mathbb{R}}\left(t+H_{p}[X, t]\right) .
$$

Some important properties and connections with other risk measures are given in Goovaerts et al. [18]. It is well known that the simplest case of the Haezendonck-Goovaerts risk measure $\mathrm{HG}_{p}[X]$ with $\phi(x)=x$ reduces to $\mathrm{CTE}_{p}[X]$. Even for a power Young function, the explicit solution to (5) is generally not available. Now, in this paper, we instead considered the asymptotic behavior of risk concentration based on the Haezendonck-Goovaerts risk measure $\mathrm{HG}_{p}[X]$ with $\phi(t)=t^{k}$ for $k \geq 1$ as $p \uparrow 1$.

Another family of risk measures, introduced by Wang [19], is defined by using the concept of the distortion function. A distortion function is an increasing function $g:[0,1] \rightarrow$ $[0,1]$ such that $g(0)=0$ and $g(1)=1$. Then for any risk $X$ with distribution function $F$, the corresponding distortion risk measure $D_{g}[\cdot]$ is defined as follows:

$$
D_{g}[X]=\int_{0}^{\infty} g(\bar{F}(t)) d t-\int_{-\infty}^{0}[1-g(\bar{F}(t))] d t
$$

where $\bar{F}(t)=1-F(t)$ denotes the survival function of $X$. The distortion risk measure has several useful properties such as positive homogeneity, translation invariance, additivity for comonotonic risks, and monotonicity. For more details, see Denuit et al. [13], Dhaene et al. [20], and Balbás et al. [21]. Several popular risk measures belong to the family of distortion risk measures. For example, the Value-at-Risk (VaR) of $X$ at the level $p$ corresponds to the distortion function $g(x)=1_{(1-p, 1]}(x), x \in[0,1]$, where $1_{E}$ is the indicator function of $E$; the conditional tail expectation (CTE) of $X$ at level $p$ corresponds to the distortion function $g(x)=\min \{x /(1-p), 1\}, x \in(0,1)$.

The tail distortion risk measure, first introduced by Zhu and $\mathrm{Li}$ [22], was reformulated by Yang [23] as follows: for a distortion function $g$, the tail distortion risk measure at level $p$ of a loss variable $X$ is defined as $T_{g_{p}}[X]=D_{g_{p}}[X], p \in$ $(0,1)$, where

$$
g_{p}(u)= \begin{cases}g\left(\frac{u}{1-p}\right), & 0 \leq u \leq 1-p, \\ 1, & 1-p<u \leq 1 .\end{cases}
$$

Since the risk is always heavy-tailed and often obeys a law of regular variation, we choose $\varrho[\cdot]$ as $\operatorname{VaR}_{p}[\cdot], \operatorname{HG}_{p}[\cdot]$, and $T_{g_{p}}[\cdot]$ at the level $0<p<1$, respectively, in (1). We denote risk concentration $C_{\varrho}$ at the level $p$ by $C_{\varrho}(p)$.

Because risk managers become more and more concerned with tail area of risk, we will focus on the secondorder approximations of the risk concentrations based on the different risk measures as $p \uparrow 1$, such as $C_{\mathrm{VaR}}(p)$, $C_{\mathrm{HG}}(p), C_{\mathrm{CTE}}(p)$, and $C_{T_{g}}(p)$ as $p \uparrow 1$ for a portfolio of $n$ loss random variables $X_{1}, X_{2}, \ldots, X_{n}$. In this paper, we assume that random variables $X_{1}, X_{2}, \ldots, X_{n}$ are identically distributed but not independent; that is,

$$
\left(X_{1}, X_{2}, \ldots, X_{n}\right)=\left(R_{1} S, R_{2} S, \ldots, R_{n} S\right),
$$

where $R_{1}, R_{2}, \ldots, R_{n}$ are $n$ i.i.d random variables with a common survival function possessing the property of secondorder regular variation, and the deflator $S$ is a random variable which is independent of $R_{1}, R_{2}, \ldots, R_{n}$.

The first-order approximations of $C_{\mathrm{VaR}}(p)$ as $p \uparrow 1$ were studied by Embrechts et al. [8,9] under the model assumption that the underlying risks $X_{1}, X_{2}, \ldots, X_{n}$ have identically distributed and regularly varying margins and have two forms of dependent structure, respectively. Degen et al. [10] derived second-order approximations of $C_{\mathrm{VaR}}(p)$ for $n$ independent and identically distributed (i.i.d) loss variables with a common survival function possessing the property of second-order regular variation (2RV). Secondorder approximations of the risk concentrations $C_{\mathrm{CTE}}(p)$ and $C_{T_{g}}(p)$ as $p \uparrow 1$ for $n$ i.i.d loss random variables were derived by Mao et al. [4], Mao and $\mathrm{Hu}$ [12], Lv et al. [6], and Hashorva et al. [7]. For a portfolio of $n$ i.i.d. risks, the second-order approximations of the risk concentrations $C_{\mathrm{VaR}}(p), C_{\mathrm{CTE}}(p)$ as $p \uparrow 1$ have been discussed by Hashorva et al. [24], while Mao and Yang [25] consider the case with a portfolio of $n$ dependent risks under FGM copula. Ling and Peng [26] derived higher-order approximations under some conditions.

The paper is organized as follows. In Section 2, we describe the definition of the second-order regular variation and some useful propositions of it. In Section 3, we obtain our main results, that is, the second-order approximations of the risk concentrations $C_{\mathrm{VaR}}(p), C_{\mathrm{HG}}(p)$, and $C_{T_{g}}(p)$ as $p \uparrow 1$, and present their proofs. In Section 4 , some examples are provided to illustrate the performance of our approximations. Throughout, the notation " $\sim$ " means asymptotic equivalence, that is, for functions $f(x)$ and $g(x)$,

$$
\begin{aligned}
f(x) & \sim g(x), \quad x \longrightarrow x_{0} \Longleftrightarrow \\
\lim _{x \rightarrow x_{0}} \frac{f(x)}{g(x)} & =1 .
\end{aligned}
$$

\section{Preliminaries}

Regular variation is one of the basic concepts which appears in different contexts of applied probability. A function $h$ is 
said to be of regular variation with index $\alpha \in \mathbb{R}$, denoted by $h \in \mathrm{RV}_{-\alpha}$, if

$$
\lim _{x \rightarrow \infty} \frac{h(x y)}{h(x)}=y^{-\alpha}
$$

holds for any $y>0$. Next we recall the definition of the second-order regular variation from de Haan and Ferreira [27] and de Haan and Stadtmüller [28]. Suppose that $h \in$ $\mathrm{RV}_{-\alpha}$ for some $\alpha \in \mathbb{R}$; then $h$ is said to be of second-order regular variation with first-order parameter $\alpha$ and secondorder parameter $\rho \leq 0$, denoted by $h \in 2 \mathrm{RV}_{-\alpha}, \rho$, if there exists some ultimately positive or negative function $A(x)$ with $A(x) \rightarrow 0$ as $x \rightarrow \infty$ such that

$$
\lim _{x \rightarrow \infty} \frac{h(x y) / h(x)-y^{-\alpha}}{A(x)}=y^{-\alpha} \int_{1}^{y} u^{\rho-1} d u, \quad \forall x>0 .
$$

Here, $A(x)$ is referred to as an auxiliary function of $h$ and $|A| \in \mathrm{RV}_{\rho}$. Several classes of parametric survival functions are shown to possess 2RV properties; see Hashorva et al. [7]. For more details on RV and 2RV, see Hua and Joe [29] and Lv et al. [5].

The function which possesses the property of secondorder regular variation (2RV) plays an important role in this article. The following proposition gives a characterization of any function $h \in 2 \mathrm{RV}_{-\alpha}, \rho$ with auxiliary function $A(x)$, $\alpha \in \mathbb{R}$ and $\rho<0$, which is from Hua and Joe [29].

Proposition 1. Let $\alpha \in \mathbb{R}, \rho<0$, and $A(x) \in R V_{\rho}$. Then $h \in 2 R V_{-\alpha}, \rho$ with auxiliary function $A(x)$ if and only if

$$
h(x)=c x^{-\alpha}\left[1+\frac{A(x)}{\rho}+o(A(x))\right], \quad x \longrightarrow \infty,
$$

where $c=\lim _{x \rightarrow \infty} x \bar{F}(x) \in(0, \infty)$.

The next two propositions give first- and second-order approximations of Haezendonck-Goovaerts risk measure $\mathrm{HG}_{p}[X]$ of $X$ at the confidence level $p$ and tail distortion risk measure $T_{g_{p}}[X]$ of $X$ at confidence level $p$ for a distortion function $g$, which will be used in the proofs of our main results.

Proposition 2. Let $X$ be a random variable with survival function $\bar{F} \in R V_{-\alpha}, \alpha>0$, and let $\phi(t)=t^{k}$ for some $\alpha>k \geq 1$. Then one has the following:

(i) The first-order asymptotic (see [30]; Mao and $\mathrm{Hu}$, 2012a):

$$
H G_{p}[X] \sim C_{\alpha} F^{\leftarrow}(p), \quad p \uparrow 1 .
$$

(ii) The second-order asymptotic (see Mao and $\mathrm{Hu}, 2012 \mathrm{a}$ ): if $\bar{F} \in 2 R V_{-\alpha, \rho}, \rho \leq 0$, with auxiliary function $A(x)$, then

$$
\begin{aligned}
& H G_{p}[X] \\
& \quad=C_{\alpha} F^{\leftarrow}(p)\left[1+H_{\alpha, \rho, k} A\left(F^{\leftarrow}(p)\right)(1+o(1))\right],
\end{aligned}
$$

where

$$
\begin{aligned}
C_{\alpha} & =\frac{\alpha(\alpha-k)^{k / \alpha-1}}{k^{k-1 / \alpha}}(B(\alpha-k, k))^{1 / \alpha}, \\
H_{\alpha, \rho, k} & =\frac{1}{\alpha \rho}\left[(\alpha-k)^{\rho k / \alpha} k^{(\rho / \alpha)(1-k)} \xi_{k, 0}^{\rho / \alpha-1} \xi_{k, \rho / \alpha}-1\right]
\end{aligned}
$$

with

$$
\xi_{k, t}=B(\alpha(1-t)-k, k)
$$

and $B(\cdot, \cdot)$ is the Beta function as usual; that is,

$$
B(a, b)=\int_{0}^{1} x^{a-1}(1-x)^{b-1} d x, \quad a, b>0 .
$$

Proposition 3. Let $X$ be a random variable with survival function $\bar{F} \in R V_{-\alpha}, \alpha>0$, and let $\mathrm{g}$ be any distortion function with

$$
\int_{0}^{1} x^{-1 / \alpha-\delta} d g(x)<\infty \quad \text { for some } \delta>0 .
$$

We have the follwoing:

(i) The first-order asymptotic (see [22, 23]):

$$
T_{g_{p}}[X] \sim J_{\alpha} F^{\leftarrow}(p), \quad p \uparrow 1 .
$$

(ii) The second-order asymptotic (see [23]): if $\bar{F} \in 2 R V_{-\alpha, \rho}$, $\rho \leq 0$, with auxiliary function $A(x)$, then

$$
\begin{aligned}
T_{g_{p}}[X]= & J_{\alpha} F^{\leftarrow}(p) \\
& +F^{\leftarrow}(p) A\left(F^{\leftarrow}(p)\right)\left[I_{\alpha, \rho}+o(1)\right],
\end{aligned}
$$

where

$$
\begin{aligned}
J_{\alpha} & =\int_{0}^{1} x^{-1 / \alpha} d g(x), \\
I_{\alpha, \rho} & =\int_{0}^{1} x^{-1 / \alpha} \frac{x^{-\rho / \alpha}-1}{\alpha \rho} d g(x) .
\end{aligned}
$$

Propositions 2(ii) and 3(ii) are, respectively, modified from Theorem 4.5 in Mao and $\mathrm{Hu}$ (2012a) and Corollary 4.1 in Yang [23] by using the fact that $\bar{F} \in 2 \mathrm{RV}_{-\alpha, \rho}$ with auxiliary function $A(t)$ if and only if its tail quantile function $U \in$ $2 \mathrm{RV}_{1 / \alpha, \rho / \alpha}$ with auxiliary function $\alpha^{-2} A(U(t))$ (see Theorem 2.3.9 in de Haan and Ferreira [27]).

\section{Main Results and Their Proofs}

3.1. Main Results. In this section, we give some results establishing the second-order approximations of the risk concentration $C_{\rho}(p)$ as $p \rightarrow 1$ for a portfolio of $n$ random variables that satisfy (9). The first one is about the risk concentration $C_{\mathrm{VaR}}(p)$. 
Theorem 4. Let $\left(X_{1}, X_{2}, \ldots, X_{n}\right)=\left(R_{1} S, R_{2} S, \ldots, R_{n} S\right)$, where $\left\{R_{1}, R_{2}, \ldots, R_{n}\right\}$ are i.i.d nonnegative random variables with common continuous distribution function $F$ and $S$ is a nonnegative random variable independent of $\left\{R_{1}, R_{2}, \ldots, R_{n}\right\}$. If $\bar{F} \in 2 R V_{-\alpha, \rho}, \alpha>0, \rho<0$, with auxiliary function $A(x)$ and $E S^{\alpha-\rho+\epsilon}<\infty$ for some $\epsilon>0$, then

(i) for $\rho<-\alpha$ and $0<\alpha<1$,

$$
\begin{aligned}
C_{V a R}(p)= & n^{1 / \alpha-1}\left[1+\frac{(n-1) \xi_{\alpha}}{2 n \alpha} \cdot \frac{E S^{2 \alpha}}{\left(E S^{\alpha}\right)^{2}}(1-p)\right] \\
& +o(1-p), \quad p \uparrow 1 ;
\end{aligned}
$$

(ii) for $\rho<-1$,

$$
\begin{aligned}
& C_{V a R}(p) \\
& =n^{1 / \alpha-1}\left[1+n^{-1 / \alpha}(n-1) \frac{E S^{\alpha+1}}{\left(E S^{\alpha}\right)^{1+1 / \alpha}} \cdot \frac{\mu_{F}}{F^{\leftarrow}(p)}\right] \\
& \quad+o\left(\frac{1}{F^{\leftarrow}(p)}\right), \quad p \uparrow 1
\end{aligned}
$$

when $\alpha>1$,

$$
\begin{aligned}
C_{V a R}(p)=1 & +c\left(1-\frac{1}{n}\right) \frac{E S^{\alpha+1}}{\left(E S^{\alpha}\right)^{1+1 / \alpha}} \\
& \cdot \frac{\log \left(F^{\leftarrow}(p)\right)}{F^{\leftarrow}(p)}(1+o(1)), \quad p \uparrow 1
\end{aligned}
$$

with $c=\lim _{x \rightarrow \infty} x \bar{F}(x) \in(0, \infty)$, when $\alpha=1$;

(iii) for $\rho>-(1 \wedge \alpha)$,

$$
\begin{aligned}
& C_{V a R}(p) \\
& =n^{1 / \alpha-1}\left[1+\frac{n^{\rho / \alpha}-1}{\alpha \rho} \cdot \frac{E S^{\alpha-\rho}}{\left(E S^{\alpha}\right)^{1-\rho / \alpha}} A\left(F^{\leftarrow}(p)\right)\right] \\
& \quad+o\left(A\left(F^{\leftarrow}(p)\right)\right), \quad p \uparrow 1 .
\end{aligned}
$$

In the following theorem, we derive the second-order asymptotic of risk concentration for Haezendonck-Goovaerts risk measure $C_{\mathrm{HG}}(p)$ at level $p$.

Theorem 5. Let $\left(X_{1}, X_{2}, \ldots, X_{n}\right)=\left(R_{1} S, R_{2} S, \ldots, R_{n} S\right)$, where $\left\{R_{1}, R_{2}, \ldots, R_{n}\right\}$ are i.i.d. nonnegative random variables with common continuous distribution function $F$ and $S$ is a nonnegative random variable independent of $\left\{R_{1}, R_{2}, \ldots, R_{n}\right\}$. If $\bar{F} \in 2 R V_{-\alpha, \rho}, \alpha>0, \rho<0$, with auxiliary function $A(x)$ and $E S^{\alpha-\rho+\epsilon}<\infty$ for some $\epsilon>0$ and if $\phi(t)=t^{k}$ for some $\alpha>k \geq 1$, then

(i) for $\rho<-\alpha$ and $0<\alpha<1$,

$$
\begin{aligned}
& C_{H G}(p)=n^{1 / \alpha-1}\left[1+\frac{n-1}{2 n \alpha} \cdot \frac{E S^{2 \alpha}}{\left(E S^{\alpha}\right)^{2}}\right. \\
&\left.\cdot\left(1-\alpha^{2} H_{\alpha,-\alpha, k}\right) \xi_{\alpha}(1-p)\right]+o(1-p), \quad p \uparrow 1 ;
\end{aligned}
$$

(ii) for $\rho<-1$,

$$
\begin{gathered}
C_{H G}(p)=n^{1 / \alpha-1}\left[1+n^{-1 / \alpha}(n-1) \frac{E S^{\alpha+1}}{\left(E S^{\alpha}\right)^{1+1 / \alpha}}\right. \\
\left.\cdot\left(1-\alpha H_{\alpha,-1, k}\right) \cdot \frac{\mu_{F}}{F^{\leftarrow}(p)}\right]+o\left(\frac{1}{F^{\leftarrow}(p)}\right),
\end{gathered}
$$

when $\alpha>1$, and

$$
\begin{aligned}
C_{H G}(p)= & 1+c\left(1-\frac{1}{n}\right) \frac{E S^{\alpha+1}}{\left(E S^{\alpha}\right)^{1+1 / \alpha}} \cdot\left(1-H_{1,-1, k}\right) \\
& \cdot \frac{\log \left(F^{\leftarrow}(p)\right)}{F^{\leftarrow}(p)}(1+o(1)), \quad p \uparrow 1
\end{aligned}
$$

with $c=\lim _{x \rightarrow \infty} x \bar{F}(x) \in(0, \infty)$ when $\alpha=1 ;$

(iii) for $\rho>-(1 \wedge \alpha)$,

$$
\begin{array}{r}
C_{H G}(p)=n^{1 / \alpha-1}\left[1+\frac{n^{\rho / \alpha}-1}{\alpha \rho} \cdot \frac{E S^{\alpha-\rho}}{\left(E S^{\alpha}\right)^{1-\rho / \alpha}}\right. \\
\left.\cdot\left(1+\alpha \rho H_{\alpha, \rho, k}\right) A\left(F^{\leftarrow}(p)\right)\right]+o\left(A\left(F^{\leftarrow}(p)\right)\right), \\
p \uparrow 1 .
\end{array}
$$

The last theorem gives the second-order asymptotic of risk concentration for tail distortion risk measure $C_{T_{g}}(p)$ at level $p$.

Theorem 6. Let $\left(X_{1}, X_{2}, \ldots, X_{n}\right)=\left(R_{1} S, R_{2} S, \ldots, R_{n} S\right)$, where $\left\{R_{1}, R_{2}, \ldots, R_{n}\right\}$ are i.i.d. nonnegative random variables with common continuous distribution function $F$, and $S$ is a nonnegative random variable independent of $\left\{R_{1}, R_{2}, \ldots, R_{n}\right\}$. Further assume that $\bar{F} \in 2 R V_{-\alpha, \rho}, \alpha>0, \rho<0$, with auxiliary function $A(x)$ and $E S^{\alpha-\rho+\epsilon}<\infty$ for some $\epsilon>0$. Let $g$ be a distortion function with

$$
\int_{0}^{1} x^{-1 / \alpha-\delta} d g(x)<\infty \quad \text { for some } \delta>0 .
$$

Then

(i) for $\rho<-\alpha$ and $0<\alpha<1$,

$$
\begin{aligned}
C_{T_{g}}(p) & =n^{1 / \alpha-1}\left[1+\frac{n-1}{2 n \alpha} \cdot \frac{E S^{2 \alpha}}{\left(E S^{\alpha}\right)^{2}}\right. \\
& \left.\left(1-\frac{\alpha^{2} I_{\alpha,-\alpha}}{J_{\alpha}}\right) \xi_{\alpha}(1-p)\right]+o(1-p), \quad p \uparrow 1 ;
\end{aligned}
$$

(ii) for $\rho<-1$,

$$
\begin{aligned}
& C_{T_{g}}(p)=n^{1 / \alpha-1}\left[1+n^{-1 / \alpha}(n-1) \frac{E S^{\alpha+1}}{\left(E S^{\alpha}\right)^{1+1 / \alpha}}\right. \\
&\left.\cdot\left(1-\frac{\alpha I_{\alpha,-1}}{J_{\alpha}}\right) \cdot \frac{\mu_{F}}{F^{\leftarrow}(p)}\right]+o\left(\frac{1}{F^{\leftarrow}(p)}\right), \quad p \uparrow 1
\end{aligned}
$$


when $\alpha>1$, and

$$
\begin{aligned}
C_{T_{g}}(p)= & 1+c\left(1-\frac{1}{n}\right) \frac{E S^{\alpha+1}}{\left(E S^{\alpha}\right)^{1+1 / \alpha}} \cdot\left(1-\frac{I_{1,-1}}{J_{1}}\right) \\
& \cdot \frac{\log \left(F^{\leftarrow}(p)\right)}{F^{\leftarrow}(p)}(1+o(1)), \quad p \uparrow 1
\end{aligned}
$$

with $c=\lim _{x \rightarrow \infty} x \bar{F}(x) \in(0, \infty)$ when $\alpha=1$;

(iii) for $\rho>-(1 \wedge \alpha)$,

$$
\begin{gathered}
C_{T_{g}}(p)=n^{1 / \alpha-1}\left[1+\frac{n^{\rho / \alpha}-1}{\alpha \rho} \cdot \frac{E S^{\alpha-\rho}}{\left(E S^{\alpha}\right)^{1-\rho / \alpha}}\right. \\
\left.\cdot\left(1+\frac{\alpha \rho I_{\alpha, \rho}}{J_{\alpha}}\right) A\left(F^{\leftarrow}(p)\right)\right]+o\left(A\left(F^{\leftarrow}(p)\right)\right),
\end{gathered}
$$

$p \uparrow 1$.

Thus, we immediately obtain the following corollary which establishes the second-order asymptotic of risk concentration for conditional tail expectation $C_{\mathrm{CTE}}(p)$. And this corollary can also be obtained easily by Lemma 8 .

Corollary 7. Let $\left(X_{1}, X_{2}, \ldots, X_{n}\right)=\left(R_{1} S, R_{2} S, \ldots, R_{n} S\right)$ be a continuous random vector, where $\left\{R_{1}, R_{2}, \ldots, R_{n}\right\}$ are i.i.d. nonnegative random variables with common continuous distribution function $F$ and $S$ is a nonnegative random variable independent of $\left\{R_{1}, R_{2}, \ldots, R_{n}\right\}$. If $\bar{F} \in 2 R V_{-\alpha, \rho}, \alpha>1, \rho<0$, with auxiliary function $A(x)$ and $E S^{\alpha-\rho+\epsilon}<\infty$ for some $\epsilon>0$. Then

(i) for $\rho<-1$,

$$
\begin{aligned}
C_{C T E}(p)= & n^{1 / \alpha-1}+\frac{n-1}{n}\left(1-\frac{1}{\alpha}\right) \cdot \frac{E S^{\alpha+1}}{\left(E S^{\alpha}\right)^{1+1 / \alpha}} \\
& \cdot \frac{\mu_{F}}{F^{\leftarrow}(p)}+o\left(\frac{1}{F^{\leftarrow}(p)}\right), \quad p \uparrow 1 ;
\end{aligned}
$$

(ii) for $\rho>-1$,

$$
\begin{gathered}
C_{C T E}(p)=n^{1 / \alpha-1}\left[1+\frac{n^{\rho / \alpha}-1}{\rho / \alpha} \cdot \frac{\alpha-1}{\alpha^{2}(\alpha-1-\rho)}\right. \\
\left.\cdot \frac{E S^{\alpha-\rho}}{\left(E S^{\alpha}\right)^{1-\rho / \alpha}} A\left(F^{\leftarrow}(p)\right)\right]+o\left(A\left(F^{\leftarrow}(p)\right)\right),
\end{gathered}
$$

$p \uparrow 1$.

3.2. Proofs. Before proving the above results, we introduce some lemmas. The first one gives a second-order form of Breiman's theorem (see Breiman [31]), which is from Hashorva et al. [7].

Lemma 8. Let $R$ be a random variable with survival function $\bar{F} \in 2 R V_{-\alpha, \rho}, \alpha>0, \rho<0$, with auxiliary function $A(x)$, and let $S$ be a nonnegative random variable satisfying $E S^{\alpha-\rho+\epsilon}<\infty$ for some $\epsilon>0$, independent of $X$. Then

$$
\frac{P(R S>x)}{\bar{F}(x)}=E S^{\alpha}[1+\varepsilon(x)]
$$

where $\varepsilon(x)=(1 / \rho)\left(E S^{\alpha-\rho} / E S^{\alpha}-1\right) A(x)(1+o(1))$ as $x \rightarrow \infty$, and thus $R S \in 2 R V_{-\alpha, \rho}$ with auxiliary function

$$
A^{*}(x)=\frac{E S^{\alpha-\rho}}{E S^{\alpha}} A(x) .
$$

The second lemma talks about the first- and second-order asymptotic of Value-at-Risk of the product $R S$ at the level $p$, which was proved by Hashorva et al. [7].

Lemma 9. Let $R$ be a random variable with survival function $\bar{F} \in R V_{-\alpha}, \alpha>0$, and let $S$ be a nonnegative random variable satisfying $E S^{\alpha+\epsilon}<\infty$ for some $\epsilon>0$, independent of $X$. Then one has the following:

(i) The first-order asymptotic:

$$
\operatorname{VaR}_{p}(R S) \sim\left(E S^{\alpha}\right)^{1 / \alpha} F^{\leftarrow}(p), \quad p \uparrow 1 .
$$

(ii) The second-order asymptotic: if $\bar{F} \in 2 R V_{-\alpha, \rho}, \rho<0$, with auxiliary function $A(x)$ and $E S^{\alpha-\rho+\epsilon}<\infty$, then

$$
\begin{array}{r}
\operatorname{VaR}_{p}(R S) \\
=\left(E S^{\alpha}\right)^{1 / \alpha} F^{\leftarrow}(p)\left[1+\zeta_{\alpha, \rho} A\left(F^{\leftarrow}(p)\right)(1+o(1))\right], \\
p \uparrow 1,
\end{array}
$$

$$
\text { where } \zeta_{\alpha, \rho}=(1 / \alpha \rho)\left(E S^{\alpha-\rho} /\left(E S^{\alpha}\right)^{1-\rho / \alpha}-1\right) \text {. }
$$

First, we introduce two definitions. Let $F$ be a distribution function of a nonnegative random variable. We introduce the truncated mean of $F$ :

$$
\mu_{F}(t)=\int_{0}^{t} x d F(x), \quad t>0
$$

Obviously, if the mean of $F, \mu_{F}$, exists, then $\mu_{F}(t) \rightarrow \mu_{F}$ as $t \rightarrow \infty$. For $0<\alpha<1$, define

$$
\xi_{\alpha}=2^{2 \alpha}-2^{\alpha+1}+2 \alpha \int_{0}^{1}\left((1-x)^{-\alpha}-1\right) x^{-\alpha-1} d x .
$$

The following lemma from $\mathrm{Mao}$ and $\mathrm{Hu}$ [12] states that the $2 \mathrm{RV}$ property is preserved by the formation of sum of $n$ i.i.d random variables.

Lemma 10. Let $F$ be the distribution function of a nonnegative random variable satisfying $\bar{F} \in 2 R V_{-\alpha, \rho}, \rho \leq 0$, with auxiliary function $A(x)$. We denoted by $F^{* n}$ the $n$-fold convolution of $F$. Then $\overline{F^{* n}} \in 2 R V_{-\alpha, \gamma}$ with auxiliary function $B(x)$, where

$$
\gamma=-\min \{1, \alpha,-\rho\},
$$


and $B(x)$ is given by

$$
\begin{aligned}
& B(x) \\
& \quad= \begin{cases}-\frac{n-1}{2} \alpha \xi_{\alpha} \bar{F}(x), & \rho<-\alpha, 0<\alpha<1, \\
-(n-1) \alpha x^{-1} \mu_{F}(x), & \rho<-1, \alpha \geq 1, \\
A(x), & \rho>-(1 \wedge \alpha) .\end{cases}
\end{aligned}
$$

The last lemma from Mao et al. [4] establishes the secondorder asymptotic of the risk concentration $C_{\mathrm{VaR}}(p)$ for $n$ i.i.d random variables with the underlying distribution possessing the $2 \mathrm{RV}$ property.

Lemma 11. Let $R_{1}, R_{2}, \ldots, R_{n}$ be i.i.d. nonnegative random variables with common continuous distribution function $F$, and assume that $\bar{F} \in 2 R V_{-\alpha, \rho}, \rho \leq 0$, with auxiliary function $A(x)$. Then

(i) for $\rho<-\alpha$ and $0<\alpha<1$,

$$
\begin{aligned}
\frac{\operatorname{VaR}_{p}\left(\sum_{i=1}^{n} R_{i}\right)}{n \operatorname{VaR}_{p}\left(R_{i}\right)}= & n^{1 / \alpha-1}\left[1+\frac{n-1}{2 n \alpha} \xi_{\alpha}(1-p)\right] \\
& +o(1-p), \quad p \uparrow 1 ;
\end{aligned}
$$

(ii) for $\rho<-1$ and $\alpha \geq 1$,

$$
\begin{aligned}
& \frac{\operatorname{VaR}_{p}\left(\sum_{i=1}^{n} R_{i}\right)}{n \operatorname{VaR}_{p}\left(R_{i}\right)} \\
& =n^{1 / \alpha-1}\left[1+n^{-1 / \alpha}(n-1) \frac{\mu_{F}\left(F^{\leftarrow}(p)\right)}{F^{\leftarrow}(p)}\right] \\
& \quad+o\left(\frac{\mu_{F}\left(F^{\leftarrow}(p)\right)}{F^{\leftarrow}(p)}\right), \quad p \uparrow 1 ;
\end{aligned}
$$

(iii) for $\rho>-(1 \wedge \alpha)$,

$$
\begin{aligned}
\frac{\operatorname{VaR}_{p}\left(\sum_{i=1}^{n} R_{i}\right)}{n \operatorname{VaR}_{p}\left(R_{i}\right)}= & n^{1 / \alpha-1}\left[1+\frac{n^{\rho / \alpha}-1}{\alpha \rho} A\left(F^{\leftarrow}(p)\right)\right] \\
& +o\left(A\left(F^{\leftarrow}(p)\right)\right), \quad p \uparrow 1 .
\end{aligned}
$$

Now we turn to prove our theorems.

Proof of Theorem 4. Define $Y=\sum_{i=1}^{n} R_{i}$, and denote by $F_{Y}$ the distribution function of $Y$. By Lemma $10, \bar{F}_{Y} \in 2 \mathrm{RV}_{-\alpha, \gamma}$ with auxiliary function $B(x)$ with $\gamma$ and $B(x)$ given by (44) and (45); we have

$$
\begin{array}{r}
\operatorname{VaR}_{p}(Y S) \\
=\left(E S^{\alpha}\right)^{1 / \alpha} F_{Y}^{\leftarrow}(p)\left[1+\zeta_{\alpha, \gamma} B\left(F_{Y}^{\leftarrow}(p)\right)(1+o(1))\right] \\
=\left(E S^{\alpha}\right)^{1 / \alpha} F_{Y}^{\leftarrow}(p)\left[1+\eta_{1}\left(F_{Y}^{\leftarrow}(p)\right)(1+o(1))\right], \\
p \uparrow 1,
\end{array}
$$

Similarly,

$$
\begin{array}{r}
\operatorname{VaR}_{p}\left(R_{i} S\right) \\
=\left(E S^{\alpha}\right)^{1 / \alpha} F^{\leftarrow}(p)\left[1+\zeta_{\alpha, \rho} A\left(F^{\leftarrow}(p)\right)(1+o(1))\right] \\
=\left(E S^{\alpha}\right)^{1 / \alpha} F^{\leftarrow}(p)\left[1+\eta_{2}\left(F^{\leftarrow}(p)\right)(1+o(1))\right], \\
p \uparrow 1,
\end{array}
$$

for $i=1,2, \ldots, n$, where $\eta_{2}(t)=\zeta_{\alpha, \rho} A(t)$ and $\left|\eta_{2}\right| \in \mathrm{RV}_{\rho}$. From Lemma 11, it follows that

$$
\frac{F_{Y}^{\leftarrow}(p)}{F^{\leftarrow}(p)} \longrightarrow n^{1 / \alpha}, \quad p \uparrow 1 .
$$

In view of $\left|\eta_{1}\right| \in \mathrm{RV}_{\gamma}$ and Theorem B.1.4 of de Haan and Ferreira [27], we have

$$
\eta_{1}\left(F_{Y}^{\leftarrow}(p)\right)=n^{\gamma / \alpha} \eta_{1}\left(F^{\leftarrow}(p)\right) \cdot(1+o(1)), \quad p \uparrow 1,
$$

where we use the fact that $B(x)$ is ultimately positive or negative. Thus,

$$
\begin{aligned}
C_{\mathrm{VaR}}(p)= & \frac{F_{Y}^{\leftarrow}(p)}{n F^{\leftarrow}(p)} \cdot \frac{1+\eta_{1}\left(F_{Y}^{\leftarrow}(p)\right)(1+o(1))}{1+\eta_{2}\left(F^{\leftarrow}(p)\right)(1+o(1))} \\
= & \frac{\operatorname{VaR}_{p}(Y)}{n \operatorname{VaR}_{p}\left(R_{i}\right)} \\
& \cdot \frac{1+n^{\gamma / \alpha} \eta_{1}\left(F^{\leftarrow}(p)\right)(1+o(1))}{1+\eta_{2}\left(F^{\leftarrow}(p)\right)(1+o(1))},
\end{aligned}
$$

Next, we consider three cases.

Case 1 ( $\rho<-\alpha$ and $0<\alpha<1$ ). In this case, from (44) and (45), it follows that $\gamma=-\alpha$ and

$$
\begin{aligned}
\eta_{1}\left(F^{\leftarrow}(p)\right) & =-\frac{(n-1) \alpha \zeta_{\alpha,-\alpha}}{2} \xi_{\alpha}(1-p) \\
& =\frac{n-1}{2 \alpha}\left(\frac{E S^{2 \alpha}}{\left(E S^{\alpha}\right)^{2}}-1\right) \xi_{\alpha}(1-p) .
\end{aligned}
$$

Note that $A\left(F^{\leftarrow}(p)\right)=o(1-p)$ as $p \uparrow 1$. So, by Lemma 11, we have

$$
\begin{aligned}
& C_{\mathrm{VaR}}(p)=\left(n^{1 / \alpha-1}\left(1+\frac{n-1}{2 n \alpha}\right) \xi_{\alpha}(1-p)\right. \\
& +o(1-p))\left(1+\frac{n-1}{2 n \alpha}\left(\frac{E S^{2 \alpha}}{\left(E S^{\alpha}\right)^{2}}-1\right) \xi_{\alpha}(1-p)\right. \\
& +o(1-p))(1+o(1))=n^{1 / \alpha-1}\left[1+\frac{(n-1) \xi_{\alpha}}{2 n \alpha}\right. \\
& \left.\quad \frac{E S^{2 \alpha}}{\left(E S^{\alpha}\right)^{2}}(1-p)\right]+o(1-p), \quad p \uparrow 1 .
\end{aligned}
$$

where $\eta_{1}(t)=\zeta_{\alpha, \gamma} B(t)$ and $\left|\eta_{1}\right| \in \mathrm{RV}_{\gamma}$. 
Case $2(\rho<-1$ and $\alpha \geq 1)$. In this case, $\gamma=-1$. By Karamata's theorem, it can be proved that $\mu_{F}(t) \in \mathrm{RV}_{0}$; see (2.7) and (2.8) in Mao and $\mathrm{Hu}(2012 \mathrm{a})$. Hence, $A(t)=o\left(\mu_{F}(t) / t\right)$ as $t \rightarrow \infty$. We have

$$
\begin{aligned}
& \eta_{1}\left(F^{\leftarrow}(p)\right)=\zeta_{\alpha,-1} B\left(F^{\leftarrow}(p)\right) \\
& =(n-1)\left(\frac{E S^{\alpha+1}}{\left(E S^{\alpha}\right)^{1+1 / \alpha}}-1\right) \frac{\mu_{F}\left(F^{\leftarrow}(p)\right)}{F^{\leftarrow}(p)} \\
& \eta_{2}\left(F^{\leftarrow}(p)\right)=\zeta_{\alpha,-1} A\left(F^{\leftarrow}(p)\right)=o\left(\frac{\mu_{F}\left(F^{\leftarrow}(p)\right)}{F^{\leftarrow}(p)}\right) .
\end{aligned}
$$

Thus,

$$
\begin{aligned}
& C_{\mathrm{VaR}}(p)=n^{1 / \alpha-1}(1 \\
& \left.+n^{-1 / \alpha}(n-1) \frac{\mu_{F}\left(F^{\leftarrow}(p)\right)}{F^{\leftarrow}(p)}(1+o(1))\right)(1 \\
& +n^{-1 / \alpha}(n-1)\left(\frac{E Y^{\alpha+1}}{\left(E Y^{\alpha}\right)^{1+1 / \alpha}}-1\right) \\
& \left.. \frac{\mu_{F}\left(F^{\leftarrow}(p)\right)}{F^{\leftarrow}(p)}(1+o(1))\right)=n^{1 / \alpha-1}[1 \\
& +n^{-1 / \alpha}(n-1) \frac{E S^{\alpha+1}}{\left(E S^{\alpha}\right)^{1+1 / \alpha}} \\
& \left.. \frac{\mu_{F}\left(F^{\leftarrow}(p)\right)}{F^{\leftarrow}(p)}(1+o(1))\right], \quad p \uparrow 1 .
\end{aligned}
$$

For $\alpha>1, \mu_{F}(t) \rightarrow \mu_{F}$ as $t \rightarrow \infty$. For $\alpha=1$, by Proposition 1 ,

$$
\begin{aligned}
\mu_{F}(t) & \sim \int_{0}^{t} \bar{F}(x) d x \sim \int_{1}^{t} c x^{-1}\left(1+\frac{A(x)}{\rho}\right) d x \\
& \sim c \log t+c \rho^{-1} \int_{1}^{t} x^{-1} A(x) d x \sim c \log t
\end{aligned}
$$

$$
t \longrightarrow \infty
$$

where the first equation follows from (28) in Mao and $\mathrm{Hu}$ [12], and the last equation follows since $\int_{1}^{\infty} x^{-1}|A(x)| d x<\infty$. Thus, we prove the case.

Case $3(\rho>-(1 \wedge \alpha))$. In this case, $\gamma=\rho$, we have

$$
\begin{aligned}
& \eta_{1}\left(F^{\leftarrow}(p)\right)=\zeta_{\alpha, \rho} A\left(F^{\leftarrow}(p)\right), \\
& \eta_{2}\left(F^{\leftarrow}(p)\right)=\zeta_{\alpha, \rho} A\left(F^{\leftarrow}(p)\right) .
\end{aligned}
$$

Thus,

$$
\begin{aligned}
& C_{\mathrm{VaR}}(p)=n^{1 / \alpha-1}\left(1+\frac{n^{\rho / \alpha}-1}{\alpha \rho} A\left(F^{\leftarrow}(p)\right)\right. \\
& \cdot(1+o(1))) \frac{1+n^{\rho / \alpha} \zeta_{\alpha, \rho} A\left(F^{\leftarrow}(p)\right)(1+o(1))}{1+\zeta_{\alpha, \rho} A\left(F^{\leftarrow}(p)\right)(1+o(1))} \\
& =n^{1 / \alpha-1}\left(1+\frac{n^{\rho / \alpha}-1}{\alpha \rho} A\left(F^{\leftarrow}(p)\right)(1+o(1))\right) \\
& \cdot\left(1+\frac{n^{\rho / \alpha}-1}{\alpha \rho}\left(\frac{E S^{\alpha-\rho}}{\left.\left(E S^{\alpha}\right)^{1-\rho / \alpha}-1\right) A\left(F^{\leftarrow}(p)\right)}\right.\right. \\
& \cdot(1+o(1)))=n^{1 / \alpha-1}\left[1+\frac{n^{\rho / \alpha}-1}{\alpha \rho} \cdot \frac{E S^{\alpha-\rho}}{\left(E S^{\alpha}\right)^{1-\rho / \alpha}}\right. \\
& \left.\cdot A\left(F^{\leftarrow}(p)\right)\right]+o\left(A\left(F^{\leftarrow}(p)\right)\right), \quad p \uparrow 1 .
\end{aligned}
$$

Proof of Theorem 5. From Proposition 2 and Lemmas 8 and 9 , we can get

$$
\begin{aligned}
& \mathrm{HG}_{p}\left[R_{i} S\right]=C_{\alpha} \operatorname{VaR}_{p}\left(R_{i} S\right) \\
& \cdot {\left[1+H_{\alpha, \rho, k} \cdot \frac{E S^{\alpha-\rho}}{\left(E S^{\alpha}\right)} A\left(\operatorname{VaR}_{p}\left(R_{i} S\right)\right)(1+o(1))\right] } \\
&= C_{\alpha} \operatorname{VaR}_{p}\left(R_{i} S\right) \\
& \cdot {\left[1+H_{\alpha, \rho, k} \cdot \frac{E S^{\alpha-\rho}}{\left(E S^{\alpha}\right)^{1-\rho / \alpha}} A\left(F^{\leftarrow}(p)\right)(1+o(1))\right], } \\
& p \uparrow 1,
\end{aligned}
$$

for $i=1,2, \ldots, n$, where we use the fact that $|A(x)| \in \mathrm{RV}_{\rho}$ and $A(x)$ is ultimately positive or negative.

Define $Y=\sum_{i=1}^{n} R_{i}$, and denote by $F_{Y}$ the distribution function of $Y$. By Lemma $10, \bar{F}_{Y} \in 2 \mathrm{RV}_{-\alpha, \gamma}$ with auxiliary function $B(x)$ with $\gamma$ and $B(x)$ given by (44) and (45). So, similarly, from Lemma 10, we can get

$$
\begin{aligned}
\mathrm{HG}_{p} & {[Y S]=C_{\alpha} \operatorname{VaR}_{p}(Y S)\left[1+H_{\alpha, \gamma, k}\right.} \\
\cdot & \left.\frac{E S^{\alpha-\gamma}}{\left(E S^{\alpha}\right)} B\left(\operatorname{VaR}_{p}(Y S)\right)(1+o(1))\right] \\
= & C_{\alpha} \operatorname{VaR}_{p}(Y S)\left[1+H_{\alpha, \gamma, k}\right. \\
\cdot & \left.\frac{E S^{\alpha-\gamma}}{\left(E S^{\alpha}\right)} B\left(\left(E S^{\alpha}\right)^{1 / \alpha} F_{Y}^{\leftarrow}(p)\right)(1+o(1))\right] \\
= & C_{\alpha} \operatorname{VaR}_{p}(Y S)\left[1+n^{\gamma / \alpha} H_{\alpha, \gamma, k}\right. \\
\cdot & \left.\frac{E S^{\alpha-\gamma}}{\left(E S^{\alpha}\right)^{1-\gamma / \alpha}} B\left(F^{\leftarrow}(p)\right)(1+o(1))\right], \quad p \uparrow 1,
\end{aligned}
$$


where we use the fact that $F_{Y}^{\leftarrow}(p) / F^{\leftarrow}(p) \rightarrow n^{1 / \alpha}$, as $p \uparrow 1, \quad$ Thus, $|B(x)| \in \mathrm{RV}_{\gamma}$ and $B(x)$ is ultimately positive or negative.

$$
\begin{aligned}
C_{\mathrm{HG}}(p) & =\frac{\mathrm{HG}_{p}[Y S]}{\sum_{i=1}^{n} \mathrm{HG}_{p}\left[R_{i} S\right]}=\frac{\operatorname{VaR}_{p}(Y S)}{n \operatorname{VaR}_{p}\left(R_{i} S\right)} \cdot \frac{\left(1+n^{\gamma / \alpha} H_{\alpha, \gamma, k} \cdot\left(E S^{\alpha-\gamma} /\left(E S^{\alpha}\right)^{1-\gamma / \alpha}\right) B\left(F^{\leftarrow}(p)\right)(1+o(1))\right)}{\left(1+H_{\alpha, \rho, k} \cdot\left(E S^{\alpha-\rho} /\left(E S^{\alpha}\right)^{1-\rho / \alpha}\right) A\left(F^{\leftarrow}(p)\right)(1+o(1))\right)} \\
& =C_{\mathrm{VaR}}(p) \cdot \frac{1+n^{\gamma / \alpha} \eta_{1}\left(F^{\leftarrow}(p)\right)(1+o(1))}{1+\eta_{2}\left(F^{\leftarrow}(p)\right)(1+o(1))},
\end{aligned}
$$

where

$$
\begin{aligned}
& \eta_{1}(x)=\frac{E S^{\alpha-\gamma}}{\left(E S^{\alpha}\right)^{1-\gamma / \alpha}} H_{\alpha, \gamma, k} B(x), \\
& \eta_{2}(x)=\frac{E S^{\alpha-\rho}}{\left(E S^{\alpha}\right)^{1-\rho / \alpha}} H_{\alpha, \rho, k} A(x) .
\end{aligned}
$$

Next, we consider three cases.

$$
\begin{aligned}
& \left.\cdot \frac{\mu_{F}\left(F^{\leftarrow}(p)\right)}{F^{\leftarrow}(p)}(1+o(1))\right)=n^{1 / \alpha-1}[1 \\
& +n^{-1 / \alpha}(n-1) \frac{E S^{\alpha+1}}{\left(E S^{\alpha}\right)^{1+1 / \alpha}} \cdot\left(1-\alpha H_{\alpha,-1, k}\right) \\
& \left.\cdot \frac{\mu_{F}\left(F^{\leftarrow}(p)\right)}{F^{\leftarrow}(p)}\right]+o\left(\frac{\mu_{F}\left(F^{\leftarrow}(p)\right)}{F^{\leftarrow}(p)}\right), \quad p \uparrow 1 .
\end{aligned}
$$

(i) For $\rho<-\alpha$ and $0<\alpha<1$. In this case, $\gamma=-\alpha$. From (45), it follows that

$$
\eta_{1}\left(F^{\leftarrow}(p)\right)=-\frac{(n-1) \alpha \xi_{\alpha}}{2} \cdot \frac{E S^{2 \alpha}}{\left(E S^{\alpha}\right)^{2}} H_{\alpha,-\alpha, k}(1-p) .
$$

Note that $A\left(F^{\leftarrow}(p)\right)=o(1-p)$ as $p \uparrow 1$. So, by Lemma 10, we have

$$
\begin{array}{r}
C_{\mathrm{HG}}(p)=n^{1 / \alpha-1}\left(1+\frac{(n-1) \xi_{\alpha}}{2 n \alpha} \cdot \frac{E S^{2 \alpha}}{\left(E S^{\alpha}\right)^{2}}(1-p)\right. \\
+o(1-p))\left(1-\frac{(n-1) \alpha \xi_{\alpha}}{2 n} \cdot \frac{E S^{2 \alpha}}{\left(E S^{\alpha}\right)^{2}}(1-p)\right. \\
+o(1-p))(1+o(1))=n^{1 / \alpha-1}\left[1+\frac{n-1}{2 n \alpha}\right. \\
\left.\cdot \frac{E S^{2 \alpha}}{\left(E S^{\alpha}\right)^{2}} \cdot\left(1-\alpha^{2} H_{\alpha,-\alpha, k}\right) \xi_{\alpha}(1-p)\right]+o(1-p), \\
p \uparrow 1 .
\end{array}
$$

(ii) $\rho<-1$ and $\alpha \geq 1$. In this case, $\gamma=-1$. By Karamata's theorem, it can be proved that $\mu_{F}(t) \in \mathrm{RV}_{0}$; see (2.7) and (2.8) in Mao and Hu (2012a). Hence, $A(t)=$ $o\left(\mu_{F}(t) / t\right)$ as $t \rightarrow \infty$. Thus,

$$
\begin{gathered}
C_{\mathrm{HG}}(p)=n^{1 / \alpha-1}\left(1+n^{-1 / \alpha}(n-1) \frac{E S^{\alpha+1}}{\left(E S^{\alpha}\right)^{1+1 / \alpha}}\right. \\
\left.. \frac{\mu_{F}\left(F^{\leftarrow}(p)\right)}{F^{\leftarrow}(p)}(1+o(1))\right) \cdot(1 \\
-n^{-1 / \alpha}(n-1) \alpha H_{\alpha,-1, k} \frac{E S^{\alpha+1}}{\left(E S^{\alpha}\right)^{1+1 / \alpha}}
\end{gathered}
$$

Considering $\mu_{F}(t)$ as $t \rightarrow \infty$ for $\alpha>1$ and $\alpha=1$ as Theorem 4 , we can get the result easily.

(iii) $\rho>-(1 \wedge \alpha)$. In this case, $\gamma=\rho$. Thus,

$$
\begin{aligned}
& C_{\mathrm{HG}}(p)=n^{1 / \alpha-1}\left(1+\frac{n^{\rho / \alpha}-1}{\alpha \rho} \cdot \frac{E S^{\alpha-\rho}}{\left(E S^{\alpha}\right)^{1-\rho / \alpha}}\right. \\
& \left.\cdot A\left(F^{\leftarrow}(p)\right)(1+o(1))\right) \cdot\left(1+\left(n^{\rho / \alpha}-1\right) H_{\alpha, \rho, k}\right. \\
& \left.\cdot \frac{E S^{\alpha-\rho}}{\left(E S^{\alpha}\right)^{1-\rho / \alpha}} A\left(F^{\leftarrow}(p)\right)(1+o(1))\right)=n^{1 / \alpha-1}[1 \\
& +\frac{n^{\rho / \alpha}-1}{\alpha \rho} \cdot \frac{E S^{\alpha-\rho}}{\left(E S^{\alpha}\right)^{1-\rho / \alpha}} \cdot\left(1+\alpha \rho H_{\alpha, \rho, k}\right) \\
& \left.\cdot A\left(F^{\leftarrow}(p)\right)\right]+o\left(A\left(F^{\leftarrow}(p)\right)\right), \quad p \uparrow 1 .
\end{aligned}
$$

Proof of Theorem 6. From Proposition 3 and Lemmas 8 and 9 , we can get

$$
\begin{array}{r}
T_{g_{p}}\left[R_{i} S\right]=J_{\alpha} \operatorname{VaR}_{p}\left(R_{i} S\right)+\operatorname{VaR}_{p}\left(R_{i} S\right) \\
\cdot A^{*}\left(\operatorname{VaR}_{p}\left(R_{i} S\right)\right)\left[I_{\alpha, \rho}+o(1)\right]=J_{\alpha} \operatorname{VaR}_{p}\left(R_{i} S\right) \\
\cdot\left[1+\frac{I_{\alpha, \rho}}{J_{\alpha}} \cdot \frac{E S^{\alpha-\rho}}{\left(E S^{\alpha}\right)^{1-\rho / \alpha}} A\left(F^{\leftarrow}(p)\right)(1+o(1))\right], \\
p \uparrow 1,
\end{array}
$$

for $i=1,2, \ldots, n$, where we use the fact that $|A(x)| \in \mathrm{RV}_{\rho}$ and $A(x)$ is ultimately positive or negative. 
Define $Y=\sum_{i=1}^{n} R_{i}$, and denote by $F_{Y}$ the distribution function of $Y$. By Lemma $10, \bar{F}_{Y} \in 2 \mathrm{RV}_{-\alpha, \gamma}$ with auxiliary function $B(x)$ with $\gamma$ and $B(x)$ given by (44) and (45). So, similarly, from Lemma 10, we can get

$$
\left.+n^{\gamma / \alpha} \frac{I_{\alpha, \gamma}}{J_{\alpha}} \cdot \frac{E S^{\alpha-\gamma}}{\left(E S^{\alpha}\right)^{1-\gamma / \alpha}} B\left(F^{\leftarrow}(p)\right)(1+o(1))\right],
$$

$$
\begin{aligned}
& T_{g_{p}}[Y S]=J_{\alpha} \operatorname{VaR}_{p}(Y S)+\operatorname{VaR}_{p}(Y S) \cdot \frac{E S^{\alpha-\rho}}{E S^{\alpha}} \\
& \cdot B\left(\operatorname{VaR}_{p}(Y S)\right)\left[I_{\alpha, \gamma}+o(1)\right]=J_{\alpha} \operatorname{VaR}_{p}(Y S)[1
\end{aligned}
$$

$$
\begin{aligned}
C_{T_{g}}(p) & =\frac{T_{g_{p}}[Y S]}{\sum_{i=1}^{n} T_{g_{p}}\left[R_{i} S\right]}=\frac{\operatorname{VaR}_{p}(Y S)}{n \operatorname{VaR}_{p}\left(R_{i} S\right)} \cdot \frac{1+n^{\gamma / \alpha}\left(I_{\alpha, \gamma} / J_{\alpha}\right) \cdot\left(E S^{\alpha-\gamma} /\left(E S^{\alpha}\right)^{1-\gamma / \alpha}\right) B\left(F^{\leftarrow}(p)\right)(1+o(1))}{1+I_{\alpha, \rho} / J_{\alpha} \cdot\left(E S^{\alpha-\rho} /\left(E S^{\alpha}\right)^{1-\rho / \alpha}\right) A\left(F^{\leftarrow}(p)\right)(1+o(1))} \\
& =C_{\mathrm{VaR}}(p) \cdot \frac{1+n^{\gamma / \alpha} \eta_{1}\left(\left(F^{\leftarrow}(p)\right)(1+o(1))\right)}{1+\eta_{2}\left(\left(F^{\leftarrow}(p)\right)(1+o(1))\right)},
\end{aligned}
$$

where

$$
\begin{aligned}
& \eta_{1}(x)=\frac{I_{\alpha, \gamma}}{J_{\alpha}} \cdot \frac{E S^{\alpha-\gamma}}{\left(E S^{\alpha}\right)^{1-\gamma / \alpha}} B(x), \\
& \eta_{2}(x)=\frac{I_{\alpha, \rho}}{J_{\alpha}} \cdot \frac{E S^{\alpha-\rho}}{\left(E S^{\alpha}\right)^{1-\rho / \alpha}} A(x) .
\end{aligned}
$$

Next, similar to Theorems 4 and 5, we consider three cases: (i) for $\rho<-\alpha$ and $0<\alpha<1$; (ii) for $\rho<-1$ and $\alpha \geq 1$; (iii) for $\rho>-(1 \wedge \alpha)$ to obtain the result. Thus, we complete the proof.

Proof of Corollary 7. Note that if the distortion function $g(x)=x$, then $T_{g, p}[X]$ reduces to $\mathrm{CTE}_{p}[X]$ for continuous risk variables $X$, and $C_{T_{g}}(p)$ reduces to $C_{\mathrm{CTE}}(p)$. It is easy to see that, for $\alpha>1$ and $\rho<0$,

$$
\begin{aligned}
J_{\alpha} & =\frac{\alpha}{\alpha-1}, \\
I_{\alpha, \rho} & =\frac{1}{(\alpha-\rho-1)(\alpha-1)},
\end{aligned}
$$

and, hence,

$$
\begin{aligned}
\frac{\alpha I_{\alpha,-1}}{J_{\alpha}} & =\frac{1}{\alpha}, \\
\frac{\alpha \rho I_{\alpha, \rho}}{J_{\alpha}} & =\frac{\alpha-1}{\alpha-1-\rho} .
\end{aligned}
$$

Therefore, the result is an immediate consequence of Theorem 6.

\section{Examples}

In this section, two examples are given to illustrate applications of our main results.

Example 1 (Burr distribution and Beta distribution). Let $R$ be a random variable with Burr distribution function $F$ given by

$$
\bar{F}(x)=\left(1+x^{-\rho}\right)^{\alpha / \rho}, \quad x>0, \alpha>0, \rho<0
$$

denoted by $F \sim \operatorname{Burr}(\alpha, \rho)$. It is known from Example 1 in Mao et al. [4] and Example 2 in Degen et al. [10] that $\bar{F} \in 2 \mathrm{RV}_{-\alpha, \rho}$ with auxiliary function $A(x)=\alpha x^{\rho}$. Suppose that $S \sim \operatorname{Beta}(a, b)$, where $\operatorname{Beta}(a, b)$ stands for the Beta distribution with positive parameters $a$ and $b$ and density function

$$
g(x)=\frac{1}{B(a, b)} x^{a-1}(1-x)^{b-1},
$$

$$
0<x<1, a, b>0 .
$$

It is obvious that $E S^{\kappa}=B(a+\kappa, b) / B(a, b)$ for all $\kappa>0$.

By Theorem 4, we have

$$
C_{\mathrm{VaR}}(p)= \begin{cases}n^{1 / \alpha-1}\left[1+\frac{(n-1) \xi_{\alpha}}{2 n \alpha} \cdot \frac{B(a, b) \cdot B(a+2 \alpha, b)}{B^{2}(a+\alpha, b)}(1-p)\right]+o(1-p) & \rho<-\alpha, 0<\alpha<1 \\ n^{1 / \alpha-1}+\frac{n-1}{n} \frac{E S^{\alpha+1}}{\left(E S^{\alpha}\right)^{1+1 / \alpha}} \cdot \mu_{F}(1-p)^{1 / \alpha}+o\left((1-p)^{1 / \alpha}\right) & \rho<-1, \alpha>1 \\ 1-\frac{n-1}{n}(1-\alpha) \log (1-\alpha)(1+o(1)) & \rho<-1, \alpha=1 \\ n^{1 / \alpha-1}\left[1+\frac{n^{\rho / \alpha}-1}{\rho} \cdot \frac{E S^{\alpha-\rho}}{\left(E S^{\alpha}\right)^{1-\rho / \alpha}}(1-p)^{-\rho / \alpha}\right]+o\left((1-p)^{-\rho / \alpha}\right) & \rho>-(1 \wedge \alpha) .\end{cases}
$$


Similarly, we can get risk concentration based on other risk measures. We set $n=2$ and compare the second-order approximations with the actual true value of $C_{\mathrm{VaR}}(p)$ for Burr distribution with different parameters $\alpha$ and $\rho$ and Beta distribution with $a=b=1$ in Figure 1 .

Example 2 (absolute student $t_{\alpha}$ distribution and Beta distribution). Let $R$ be a random variable having the standard Student $t_{\alpha}$ distribution with density function

$$
f_{R}(r)=\frac{\Gamma((\alpha+1) / 2)}{\sqrt{\alpha \pi} \Gamma(\alpha / 2)}\left(1+\frac{r^{2}}{\alpha}\right)^{-(\alpha+1) / 2}, \quad r>0 .
$$

Denote by $F$ the distribution function of $R$. Suppose that $S \sim$ $\operatorname{Beta}(a, b)$, where $\operatorname{Beta}(a, b)$ stands for the Beta distribution with positive parameters $a$ and $b$ and density function

$$
\begin{aligned}
g(x)=\frac{1}{B(a, b)} x^{a-1}(1-x)^{b-1}, & \\
& 0<x<1, a, b>0 .
\end{aligned}
$$

From Example 3 in Hua and Joe [29], we know that $\bar{F} \in$ $2 \mathrm{RV}_{-\alpha,-2}$ and the mean of $F, \mu_{F}=\alpha /(\alpha-1)$ for $\alpha>1$ and $E S^{\kappa}=B(a+\kappa, b) / B(a, b)$ for all $\kappa>0$.

For $0<\alpha<1$, by Theorems 4(i) and 3.2(i), we have

$$
\begin{gathered}
C_{\mathrm{VaR}}(p)=n^{1 / \alpha-1}\left[1+\frac{(n-1) \xi_{\alpha}}{2 n \alpha}\right. \\
\left.. \frac{B(a, b) \cdot B(a+2 \alpha, b)}{B^{2}(a+\alpha, b)}(1-p)\right]+o(1-p), \\
C_{\mathrm{HG}}(p)=n^{1 / \alpha-1}\left[1+\frac{n-1}{2 n \alpha} \cdot \frac{B(a, b) \cdot B(a+2 \alpha, b)}{B^{2}(a+\alpha, b)}\right. \\
\left.\cdot\left(1-\alpha^{2} H_{\alpha,-\alpha, k}\right) \xi_{\alpha}(1-p)\right]+o(1-p), \quad p \uparrow 1 .
\end{gathered}
$$

Choose distortion function $g(x)=x^{1 / \beta}$ with $0<\beta<\alpha$. It is easy to see that

$$
\begin{aligned}
J_{\alpha} & =\frac{\alpha}{\alpha-\beta}, \\
I_{\alpha,-1} & =\frac{\beta}{\alpha(\alpha-\beta)}, \\
I_{\alpha,-\alpha} & =\frac{\beta}{(\alpha-\beta)(\alpha-\beta+\alpha \beta)} .
\end{aligned}
$$

By Theorem 6(i), we have

$$
\begin{gathered}
C_{T_{g}}(p)=n^{1 / \alpha-1}\left[1+\frac{n-1}{2 n \alpha} \cdot \frac{B(a, b) \cdot B(a+2 \alpha, b)}{B^{2}(a+\alpha, b)}\right. \\
\left.\cdot \frac{\alpha-\beta}{\alpha-\beta+\alpha \beta} \xi_{\alpha}(1-p)\right]+o(1-p), \quad p \uparrow 1 .
\end{gathered}
$$

For $\alpha>1$, since $F^{\leftarrow}(p)=t_{\alpha}^{\leftarrow}((1+p) / 2)$, the quantile function of $t_{\alpha}$ distribution is at the level $(p+1) / 2$. By Theorems 4(i) and 3.2(i), we have that, as $p \uparrow 1$,

$$
\begin{aligned}
& C_{\mathrm{VaR}}(p)=n^{1 / \alpha-1}[1 \\
& +n^{-1 / \alpha}(n-1) \frac{(B(a, b))^{1 / \alpha} \cdot B(a+\alpha+1, b)}{(B(a+\alpha, b))^{1+1 / \alpha}} \\
& \left.\cdot \frac{\alpha}{\alpha-1} \cdot \frac{1}{t_{\alpha}^{\leftarrow}((1+p) / 2)}\right]+o\left(\frac{1}{t_{\alpha}^{\leftarrow}((1+p) / 2)}\right) \\
& C_{\mathrm{HG}}(p)=n^{1 / \alpha-1}[1 \\
& +n^{-1 / \alpha}(n-1) \frac{(B(a, b))^{1 / \alpha} \cdot B(a+\alpha+1, b)}{(B(a+\alpha, b))^{1+1 / \alpha}} \\
& \left.\quad . \frac{\alpha\left(1-\alpha H_{\alpha,-1, k}\right)}{\alpha-1} \cdot \frac{1}{t_{\alpha}^{\leftarrow}((1+p) / 2)}\right] \\
& +o\left(\frac{1}{t_{\alpha}^{\leftarrow}((1+p) / 2)}\right) .
\end{aligned}
$$

Considering the distortion function $g(x)=x^{1 / \beta}$ with $0<\beta<$ $\alpha$, by Theorem 6(ii), we have that, as $p \uparrow 1$,

$$
\begin{aligned}
& C_{T_{g}}(p)=n^{1 / \alpha-1}[1 \\
& +n^{-1 / \alpha}(n-1) \frac{(B(a, b))^{1 / \alpha} \cdot B(a+\alpha+1, b)}{(B(a+\alpha, b))^{1+1 / \alpha}} \\
& \left.\quad \cdot \frac{\alpha-\beta}{\alpha-1} \cdot \frac{1}{t_{\alpha}^{\leftarrow}((1+p) / 2)}\right]+o\left(\frac{1}{t_{\alpha}^{\leftarrow}((1+p) / 2)}\right) .
\end{aligned}
$$

Choosing $\beta=1, C_{T_{g}}(p)$ reduces to

$$
\begin{array}{r}
C_{\mathrm{CTE}}(p)=n^{1 / \alpha-1}[1 \\
+n^{-1 / \alpha}(n-1) \frac{(B(a, b))^{1 / \alpha} \cdot B(a+\alpha+1, b)}{(B(a+\alpha, b))^{1+1 / \alpha}} \\
\left.\cdot \frac{1}{t_{\alpha}^{\leftarrow}((1+p) / 2)}\right]+o\left(\frac{1}{t_{\alpha}^{\leftarrow}((1+p) / 2)}\right),
\end{array}
$$

We set $n=2$ and compare the second-order approximations with the actual true value of $C_{\mathrm{VaR}}(p)$ for $t_{\alpha}$ distribution with different parameters $\alpha$ and Beta distribution with $a=$ $b=1$ in Figure 2 .

\section{Conflicts of Interest}

The authors declare that they have no conflicts of interest. 

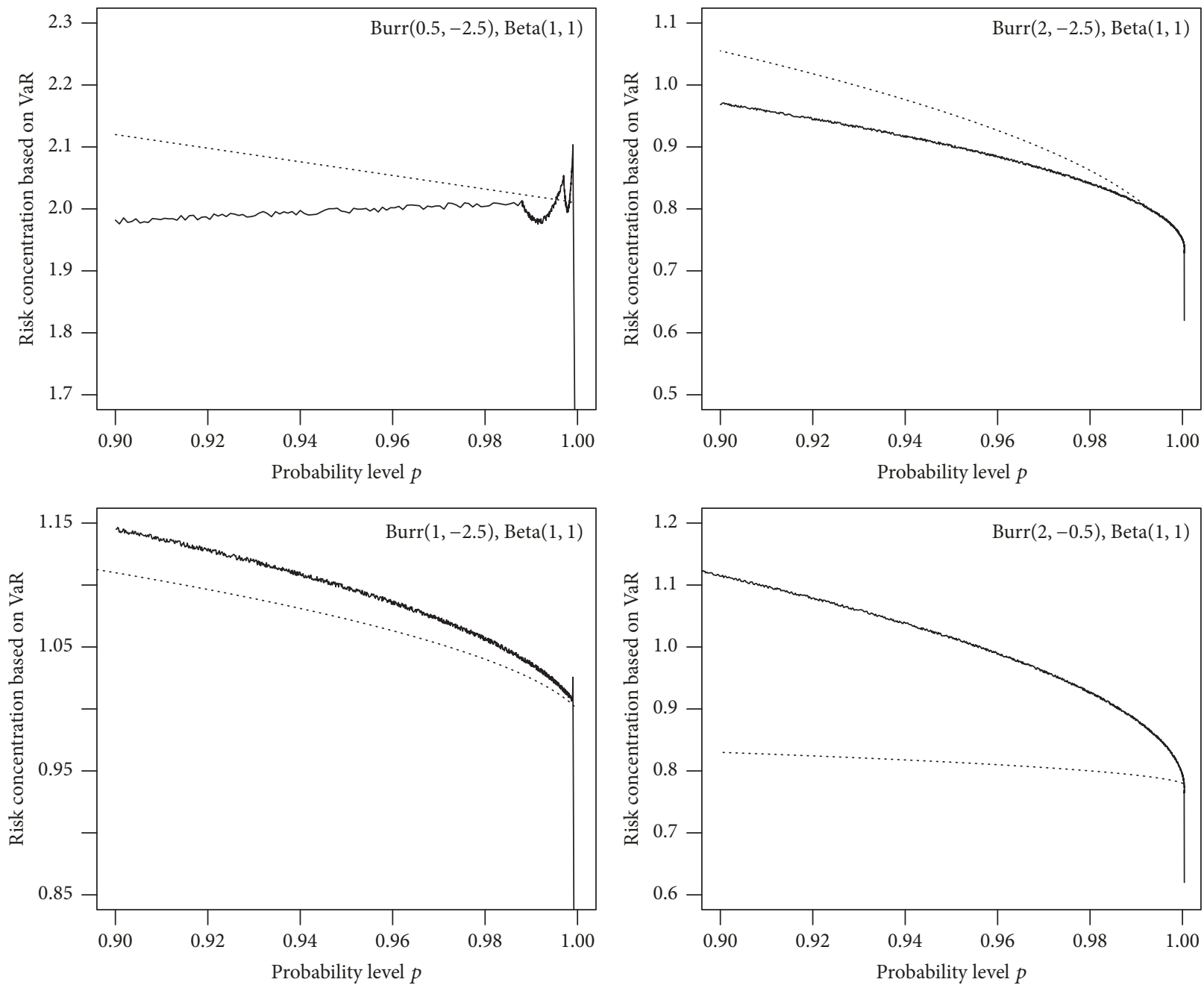

FIGURE 1: Empirical risk concentration (full, based on $10^{7}$ simulations) together with second-order approximation (dotted) for two i.i.d. $\operatorname{Burr}(\alpha, \rho)$ and $\operatorname{Beta}(1,1)$ random variables based on $C_{\mathrm{VaR}}(p)$.

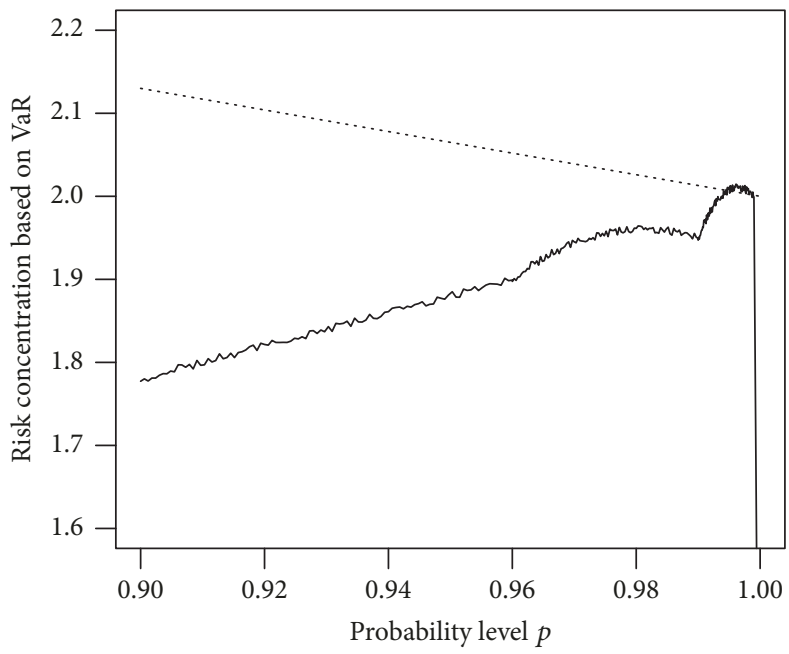

(a)

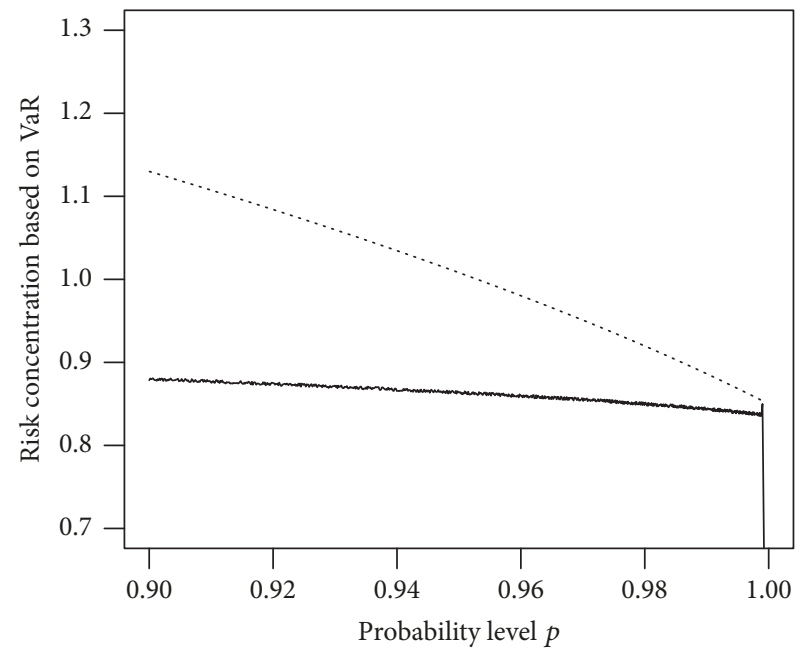

(b)

FIGURE 2: Empirical risk concentration (full, based on $10^{7}$ simulations) together with second-order approximation (dotted) for two i.i.d. $t_{\alpha}$ and $\operatorname{Beta}(1,1)$ random variables based on $C_{\mathrm{VaR}}(p)$ with $\alpha=0.5$ for $(\mathrm{a})$ and $\alpha=1.4$ for (b). 


\section{Acknowledgments}

The work is supported by the National Key Research and Development Plan (no. 2016YFC0800104) and NSFC (nos. 71771203 and 11671374).

\section{References}

[1] M. Degen, P. Embrechts, and D. D. Lambrigger, "The quantitative modeling of operational risk: Between G-and-H and EVT," ASTIN Bulletin, vol. 37, no. 2, pp. 265-291, 2007.

[2] R. Ibragimov and J. Walden, "The limits of diversification when losses may be large," Journal of Banking \& Finance, vol. 31, no. 8, pp. 2551-2569, 2007.

[3] R. Ibragimov, D. Jaffee, and J. Walden, "Nondiversification traps in catastrophe insurance markets," Review of Financial Studies, vol. 22, no. 3, pp. 959-993, 2009.

[4] T. Mao, W. Lv, and T. Hu, "Second-order expansions of the risk concentration based on CTE," Insurance: Mathematics \& Economics, vol. 51, no. 2, pp. 449-456, 2012.

[5] W. Lv, T. Mao, and T. Hu, "Properties of second-order regular variation and expansions for risk concentration," Probability in the Engineering and Informational Sciences, vol. 26, no. 4, pp. 535-559, 2012.

[6] W. Lv, X. Pan, and T. Hu, "Asymptotics of the risk concentration based on the tail distortion risk measure," Statistics \& Probability Letters, vol. 83, no. 12, pp. 2703-2710, 2013.

[7] E. Hashorva, C. Ling, and Z. Peng, "Second-order tail asymptotics of deflated risks," Insurance: Mathematics \& Economics, vol. 56, pp. 88-101, 2014.

[8] P. Embrechts, D. D. Lambrigger, and M. V. Wüthrich, "Multivariate extremes and the aggregation of dependent risks: examples and counter-examples," Extremes, vol. 12, no. 2, pp. 107-127, 2009.

[9] P. Embrechts, J. Nešlehová, and M. V. Wūthrich, "Additivity properties for value-at-risk under Archimedean dependence and heavy-tailedness," Insurance: Mathematics \& Economics, vol. 44, no. 2, pp. 164-169, 2009.

[10] M. Degen, D. D. Lambrigger, and J. Segers, "Risk concentration and diversification: second-order properties," Insurance: Mathematics \& Economics, vol. 46, no. 3, pp. 541-546, 2009.

[11] T. Mao and T. Hu, "Second-order properties of the Haezendonck-Goovaerts risk measure for extreme risks," Insurance: Mathematics \& Economics, vol. 51, no. 2, pp. 333-343, 2012.

[12] T. Mao and T. Hu, "Second-order properties of risk concentrations without the condition of asymptotic smoothness," Extremes, vol. 16, no. 4, pp. 383-405, 2013.

[13] M. Denuit, J. Dhaene, M. Goovaerts, and R. Kaas, Actuarial Theory for Dependent Risks: Measures, Orders and Models, John Wiley \& Sons, Hoboken, NJ, USA, 2005.

[14] P. Artzner, F. Delbaen, J.-M. Eber, and D. Heath, "Coherent measures of risk," Mathematical Finance, vol. 9, no. 3, pp. 203228, 1999.

[15] K. C. Cheung and A. Lo, "General lower bounds on convex functionals of aggregate sums," Insurance: Mathematics \& Economics, vol. 53, no. 3, pp. 884-896, 2013.

[16] Y. Zhu, L. Zhang, and Y. Zhang, "Optimal reinsurance under the Haezendonck risk measure," Statistics \& Probability Letters, vol. 83, no. 4, pp. 1111-1116, 2013.

[17] J. Haezendonck and M. Goovaerts, "A new premium calculation principle based on Orlicz norms," Insurance: Mathematics \& Economics, vol. 1, no. 1, pp. 41-53, 1982.
[18] M. Goovaerts, D. Linders, K. Van Weert, and F. Tank, “On the interplay between distortion, mean value and HaezendonckGoovaerts risk measures," Insurance: Mathematics \& Economics, vol. 51, no. 1, pp. 10-18, 2012.

[19] S. Wang, "Premium calculation by transforming the layer premium density," ASTIN Bulletin, vol. 26, no. 1, pp. 71-92, 1996.

[20] J. Dhaene, S. Vanduffel, M. J. Goovaerts, R. Kaas, Q. Tang, and D. Vyncke, "Risk measures and comonotonicity: a review," Stochastic Models, vol. 22, no. 4, pp. 573-606, 2006.

[21] A. Balbás, J. Garrido, and S. Mayoral, "Properties of distortion risk measures," Methodology and Computing in Applied Probability, vol. 11, no. 3, pp. 385-399, 2009.

[22] L. Zhu and H. Li, "Tail distortion risk and its asymptotic analysis," Insurance: Mathematics \& Economics, vol. 51, no. 1, pp. 115121, 2012.

[23] F. Yang, "First- and second-order asymptotics for the tail distortion risk measure of extreme risks," Communications in Statistics-Theory and Methods, vol. 44, no. 3, pp. 520-532, 2015.

[24] E. Hashorva, C. Ling, and Z. Peng, "Tail asymptotic expansions for L-statistics," Science China Mathematics, vol. 57, no. 10, pp. 1993-2012, 2014.

[25] T. Mao and F. Yang, "Risk concentration based on expectiles for extreme risks under FGM copula," Insurance: Mathematics \& Economics, vol. 64, pp. 429-439, 2015.

[26] C. Ling and Z. Peng, "Tail asymptotics of generalized deflated risks with insurance applications," Insurance: Mathematics \& Economics, vol. 71, pp. 220-231, 2016.

[27] L. de Haan and A. Ferreira, Extreme Value Theory: An Introduction. Springer Series in Operations Research and Financial Engineering, Springer, New York, NY, USA, 2006.

[28] L. de Haan and U. Stadtmüller, "Generalized regular variation of second order," Journal of the Australian Mathematical Society: Pure Mathematics and Statistics. Series A, vol. 61, no. 3, pp. 381395, 1996.

[29] L. Hua and H. Joe, "Second order regular variation and conditional tail expectation of multiple risks," Insurance: Mathematics \& Economics, vol. 49, no. 3, pp. 537-546, 2011.

[30] Q. Tang and F. Yang, "On the Haezendonck-Goovaerts risk measure for extreme risks," Insurance: Mathematics \& Economics, vol. 50, no. 1, pp. 217-227, 2012.

[31] L. Breiman, "On some limit theorems similar to the arc-sin law," Theory of Probablity and Its Application, vol. 10, pp. 323-331, 2006. 


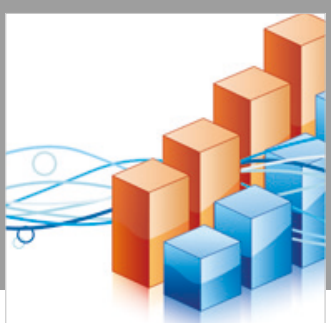

Advances in

Operations Research

\section{-n-m}
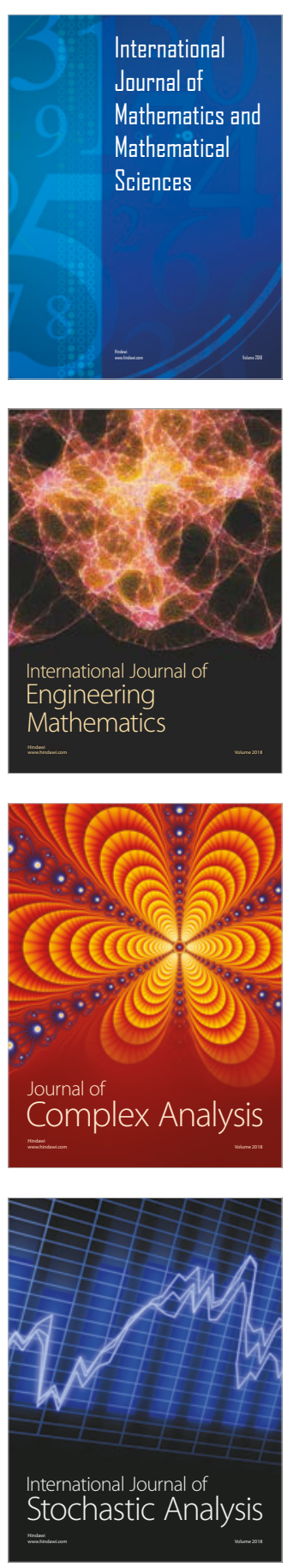
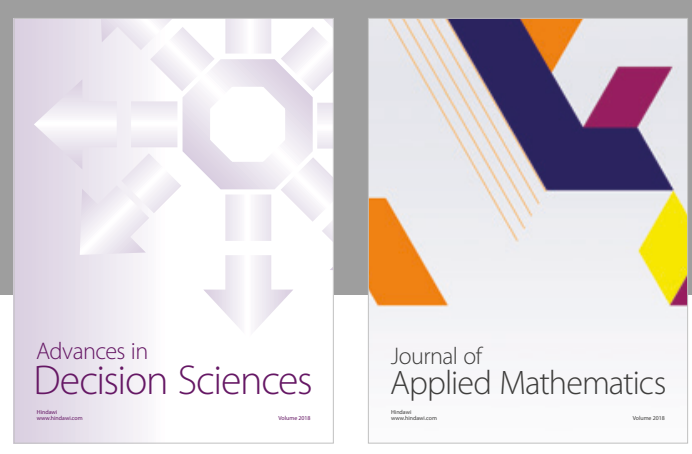

Journal of

Applied Mathematics
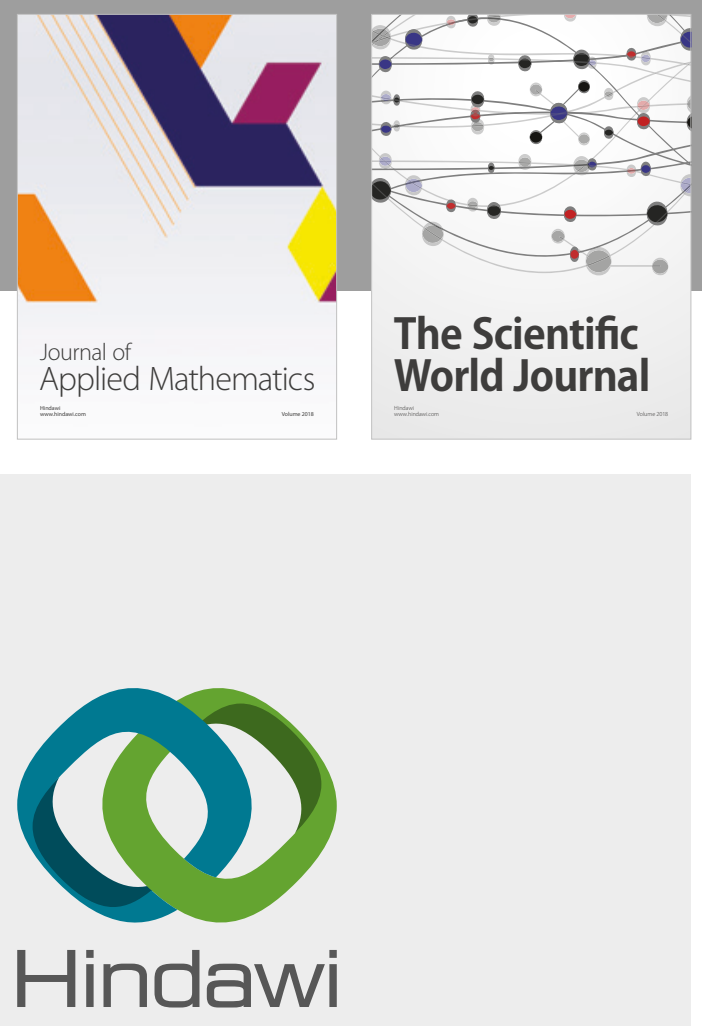

Submit your manuscripts at

www.hindawi.com

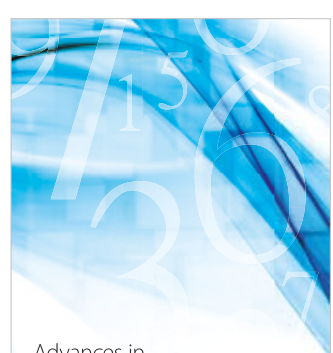

Advances in
Numerical Analysis
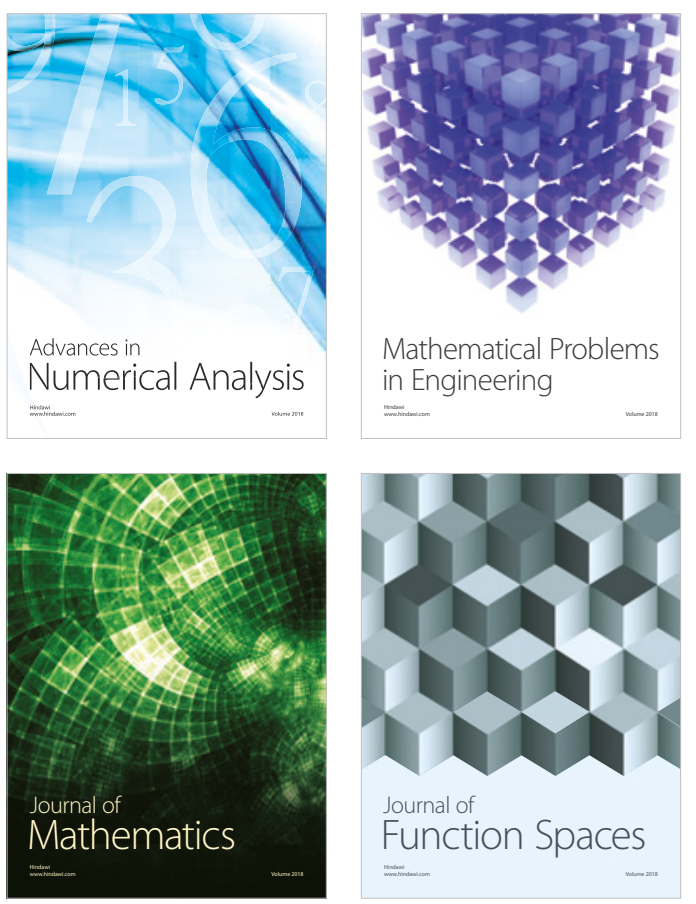

Mathematical Problems in Engineering

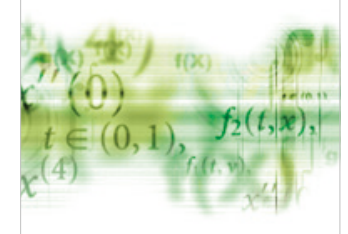

International Journal of

Differential Equations

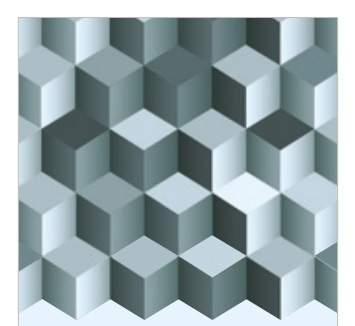

Journal of

Function Spaces

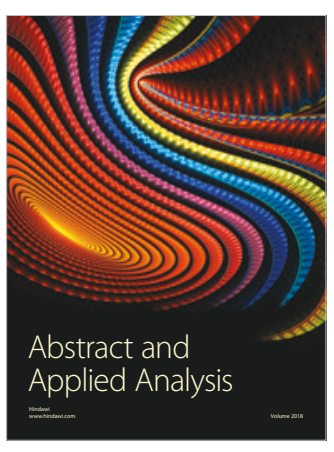

The Scientific

World Journal

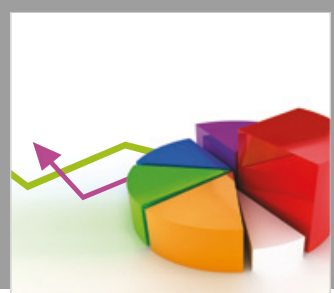

Journal of

Probability and Statistics
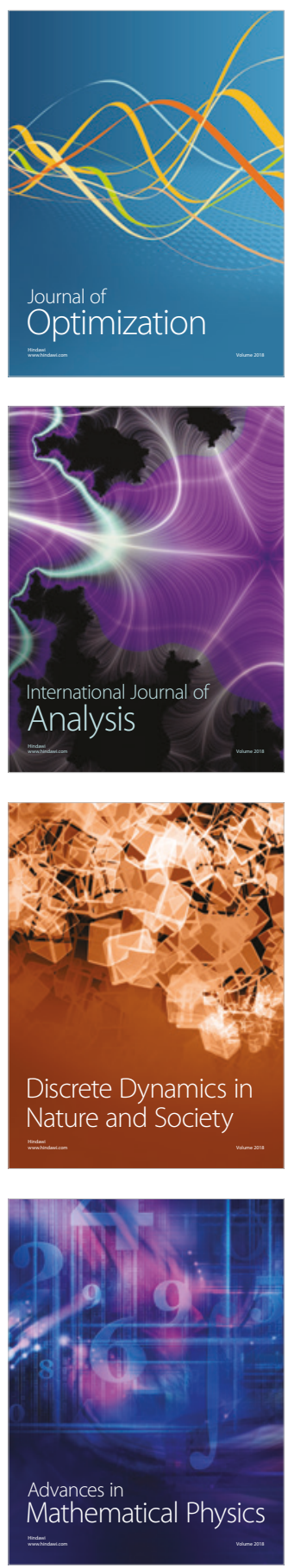